\title{
Signal Processing, Wavelets and High-speed Image Interpretation of Bird Impact
}

\author{
K. Karthikeyan*, S. Ramachandra*, S. Paul Vizhian ${ }^{\dagger}$, and Satish Chandra* \\ *National Aerospace Laboratory, Bengaluru-560 017 \\ "Gas Turbine Research Establishment, Bengaluru-560 093 \\ ${ }^{\dagger}$ University Visvesvaraya College of Engineering, Bengaluru-560 001 \\ E-mail: ram.gtre@gmail.com
}

\begin{abstract}
Bird impact on aircraft has been well documented and has been of interest for researchers in aircraft design. The process, though of very short duration, is complex in nature. A bird, which can be treated as a soft body behaves more like a fluid at high-speeds. When aircraft components become targets of bird strikes, the impact can have consequences for safety, and hence the study of the phenomena has engineering implications. In this paper, strain signals from a specially instrumented stiff fixture, high-speed imaging and wavelets are used to describe the nature of the phenomenon. Gelatin-based artificial birds were impacted on the fixture fired through an air gun at two different velocities. high-speed imaging showed different behaviours with a rebound at low-velocity $(\sim 50 \mathrm{~m} / \mathrm{s})$ and a flow behaviour at high-velocity $(\sim 100 \mathrm{~m} / \mathrm{s})$. High sampling data acquisition was used to measure the dynamic strain exerted on the fixture during bird impact. Time histories of strain signals obtained in the raw form were processed to get a Fourier spectrum and continuous wavelet transform to gain more information about different frequency patterns and the temporal distribution of the frequencies, when such impacts occurred. The frequency content for low-velocity and high-velocity impacts is characterised. It can be noted that the behaviour as described by earlier researchers was seen here as well at higher velocities, though at lower velocities, the bird behaved more like a solid. Many aircraft have approach speeds that are about $60 \mathrm{~m} / \mathrm{s}$ rather than $100-200 \mathrm{~m} / \mathrm{s}$, making it important to study behaviour at lower velocities as well. The short time interval events identified in the signals provide insight into the nature of the loads on targets. This information can aid in tuning simulation models of the birds which use Lagrangian, Eulerian and smooth particle hydrodynamic models.
\end{abstract}

Keywords: Bird impact, image processing, signal processing, wavelets, simulation modules

\section{INTRODUCTION}

Bird strikes on aircraft have been a long standing problem, with serious consequences for safety. Aircraft wings, horizontal tail leading edges, windshield, canopy and supporting structures have proved to be particularly vulnerable to bird strike impact. These impacts have at various times caused serious accidents, sometimes resulting in fatalities. Regulatory requirements ${ }^{1}$ are available for the design of these components. However, the phenomena associated with bird impact and the load generated is a matter of research ${ }^{2-4}$. At present, several types of simulation models, such as Lagrangian, Eulerian and smooth particle hydrodynamics (SPH), are used to model and predict bird impact behaviour. A detailed understanding of the behaviour of the bird is essential to refine the simulation models and enhance their usefulness ${ }^{5-12}$. Bird strikes are generally extremely short duration events that last only a few milliseconds. Wilbeck and co-workers ${ }^{2-4}$ studied the characterization of bird impact on rigid surfaces by launching small birds with velocities ranging from $100 \mathrm{~m} / \mathrm{s}$ to $350 \mathrm{~m} / \mathrm{s}$ and measuring consequent pressure data. The time histories of pressure were described as a relatively low frequency 'base pressure', on which a high frequency pressure variation was superimposed, as illustrated in Fig. 1.

It is to be noted that Barber and Wilbeck ${ }^{2}$ made only a qualitative representation. In this paper, this figure has been incorporated to emphasise the complex nature of the behaviour. The base pressure profile remained similar from shot to shot, although amplitude and duration varied with velocity as well as with bird size. The high frequency component appeared to have little repeatable structure. Wilbeck ${ }^{2}$, et al. have attributed high frequency components in the signal to real bird impact and have suggested that a high frequency signal component be regarded as a particular and real characteristic of bird impact. A bird strike, in cases where the impacts loads are transmitted, can cause local damage at the point of impact as well as at support locations. Therefore, one needs to clearly understand the frequency components in the signal and their duration. It was found that the peak pressure varied based on the velocity and was independent of bird size and it was suggested that the bird behaves like a fluid during impact ${ }^{4}$.

An experimental and analytical investigation was carried out by Barber ${ }^{3}$, et al at velocities above $100 \mathrm{~m} / \mathrm{s}$ to study 


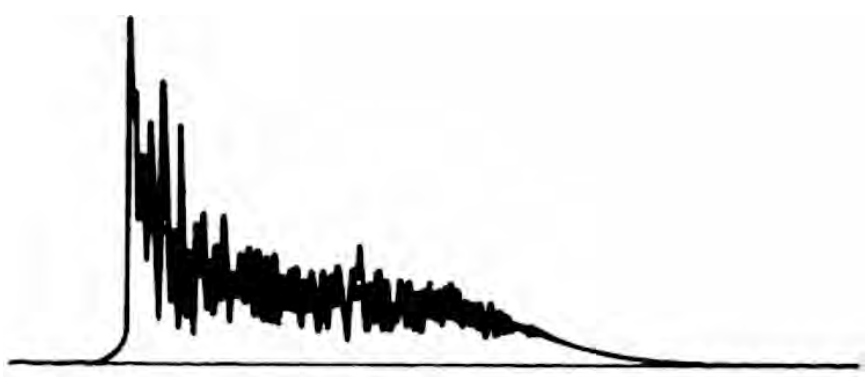

(a) Typical pressure transducer output

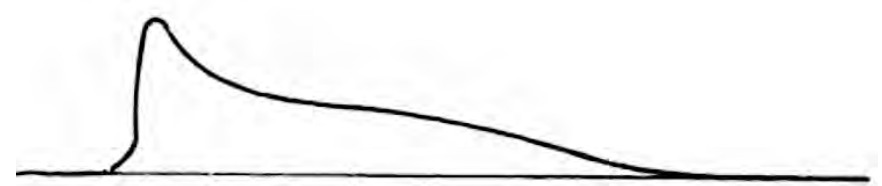

(b) Base component of pressure transducer output

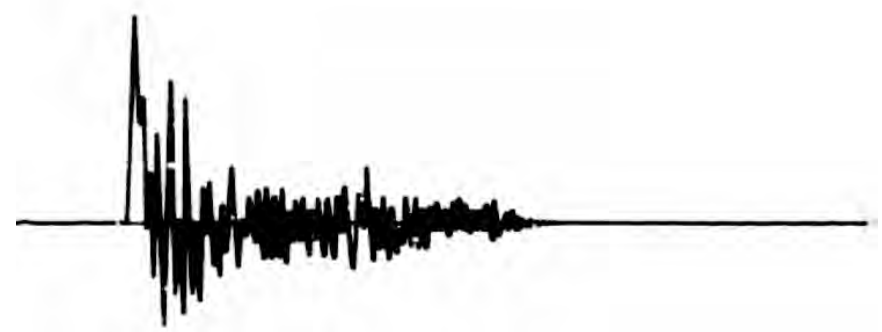

(c) High frequency pressure variation component

Figure 1. Typical components of impact pressure for real bird impact $^{2}$.

the bird impact forces and pressures on rigid and compliant targets. It was found that the principal constituent of flesh is water and it was noted that presence of porosity was an important component responsible for the behaviour of real birds on impact. A good equivalent material model was found to be gelatin with an initial density of $1.05 \mathrm{~g} /$ $\mathrm{cm}^{3}$ and porosity of 10 per cent.

There are four distinct regimes of fluid flow when a cylindrical dummy bird impacts a rigid $\operatorname{target}^{3}$ (as shown in Fig. 2). The first phase is the initial impact in which very high shock or Hugoniot pressures are generated. The second phase involves the decay of those very high shock pressures down to steady fluid flow pressures. The third phase is the phase in which the bird material flows steadily onto the target and an equivalent jet flow is established. The fourth and last phase involves termination of the impact process and the return of the impact force and pressures to zero. Birds were found to behave like fluids during impact ${ }^{2}$. The flow can be assumed as one-dimensional, adiabatic, and irreversible. The shock pressure is a function of shock velocity, the impact velocity, and bird density.

It was also noted that the shock was weakened by the release waves in this phase and would be ultimately eliminated. After several reflections, a condition of steady flow is established and steady pressures obtained. It is thus useful to investigate this via high-speed imaging and measurement of response. Further, based on the empirical

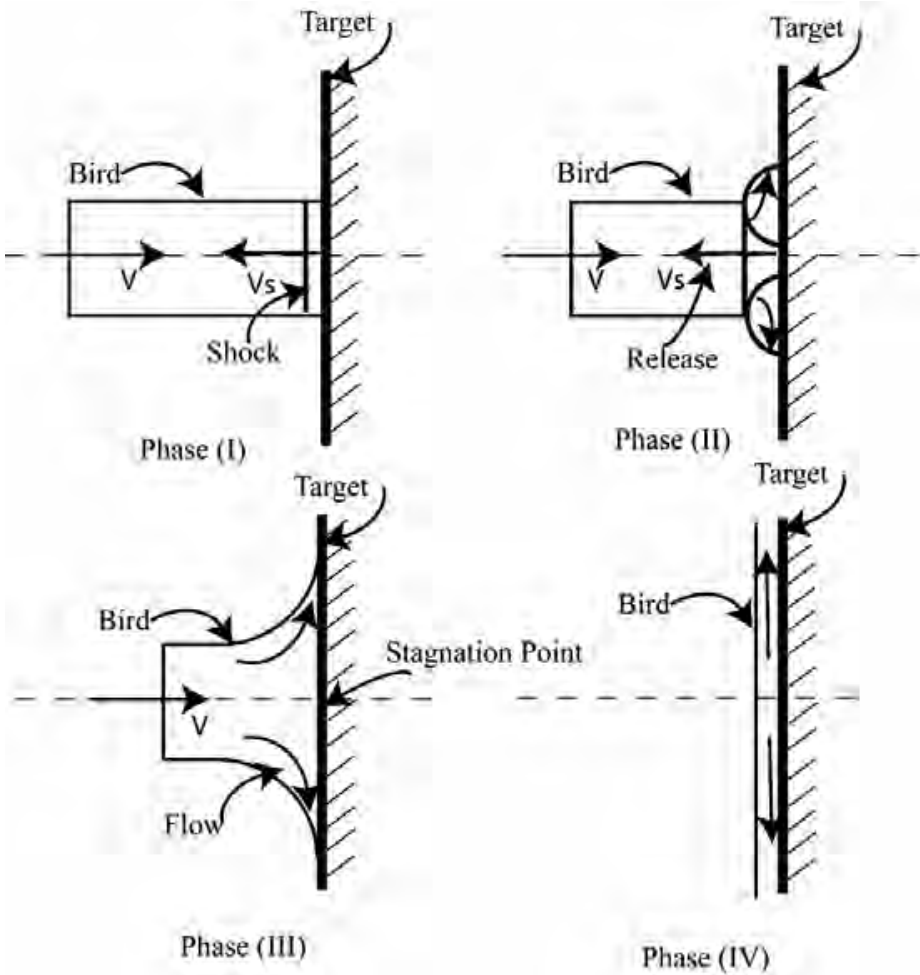

Figure 2. Different stages of bird impact ${ }^{3}$.

relationship between the time duration of impact and velocity and length of the bird, the different phases and duration of such phases was estimated. Wilbeck ${ }^{2}$ computed shock pressures for various velocities and found it to be very high (100-500 MN/m²) for velocities between $150-350 \mathrm{~m} /$ $\mathrm{s}$. It is thus important to understand the range of frequencies these shock pressures activate. Radial pressures decrease during the shock pressure decay and shear stress develops in the projectile materials. It was hypothesised that if the shear strength of the material was sufficient to withstand these shear stresses, the radial motion of the projectile was be restricted. If however, the shear stresses of the material exceed its shear strength, the material will flow like a fluid. A high-speed image will facilitate the visualisation of these events, which have been studied.

To describe the process of bird impact, an unsteady, hydrodynamic model of the impact of a low-strength projectile on a rigid target has been reported by Wilbeck ${ }^{4}$. This theoretical model was verified with experimental test results. Wilbeck found that the impact of any material whose shock pressure generated during impact is much greater than the strength of the projectile, the material will flow. This condition explains the nature of the low-strength projectile during the impact at high-speeds. However, at lower speeds, it is possible that the bird does not behave like a fluid fully as the shock pressures are lower. Instead, the behaviour could be considered as a solid material with exact material properties that need to be worked out.

Brockman ${ }^{5}$ reported that when the structure is relatively flexible, the soft body impact involves strong coupling. McNaughtan $^{6}$ investigated the bird impact resistance of 
aircraft leading edge structures. He has derived empirical design formulae for penetration velocity of flat and curved panels and leading edge structures. The design formulae have been verified with experimental results.

Many researchers have studied the bird impact behaviour through test and analysis on the various targets such as composites, sandwich panels, aero-engines and glass panels ${ }^{4-}$ ${ }^{13}$. It is important to study the bird behaviour to improve bird strike-resistant design. In this study, emphasis is given to low-velocity impact $(50-60 \mathrm{~m} / \mathrm{s})$ which doesn't confirm fully with the four-stage hydrodynamic theory proposed by Wilbeck ${ }^{3}$. To ascertain if the bird behaves as a fluid or solid and to understand if rebound occurs, the behaviour of the bird needs to be investigated at lower $(50-60 \mathrm{~m} / \mathrm{s})$ and higher $(90-120 \mathrm{~m} / \mathrm{s})$ velocities. To achieve this, high-speed imaging, signal processing, and wavelet analysis, that also enables estimation of the temporal distribution of frequencies, are used.

\section{EXPERIMENTAL SETUP}

A series of bird strike tests were conducted to measure the strain-time histories on the test fixture at the Gas Turbine Research Establishment (GTRE) Bird Ingestion Test Facility. A schematic illustration of the experimental set-up used for the present study is shown in Fig. 3. The facility essentially consists of an air gun with a $12 \mathrm{~m}$ long gun barrel, an air reservoir, and a breach mechanism. The dummy bird projectile is assembled in a steel canister with foam packing. The projectile is arrested at the end of the gun barrel releasing only the projectile on to the target. The target fixture is rigidly mounted on a base. Strain gages are mounted on the test fixture. A high-speed camera along with adequate lighting arrangement is used to record the event.

The target fixture as shown in Fig. 4 is basically a steel plate welded to a cylindrical hub which is then subsequently welded to a circular plate. This plate is bolted to a parent mount that is fixed to a steel wall. The hub is strain gauged as noted earlier.

Four strain gauges were mounted along the circumference of the cylindrical hub. The measured signals were conditioned by subsequently passing through a $25 \mathrm{kHz}$ anti-aliasing filter and recorded at a rate of $50 \mathrm{kHz}$ sampling frequency by a digital data acquisition system (Prosig Model P8020). The bird behaviour was viewed and captured using a high-speed digital camera (Redlake Inc, US). The camera has a CMOS sensor technology with capability up to 1000 frames per second (fps) at full (1024 X 1024) resolution. However, the present impact events are recorded at 2500 fps with reduced resolution.

The two-pound dummy bird projectiles made of gelatine were accelerated by means of the single-stage air gun. The bird density was varied from $0.92 \mathrm{~g} / \mathrm{cc}$ to $1.11 \mathrm{~g} / \mathrm{cc}$ and the behaviour was categorised as low $(0.92 \mathrm{~g} / \mathrm{cc})$, medium $(0.9400 \mathrm{~g} / \mathrm{cc})$ and high $(1.110 \mathrm{~g} / \mathrm{cc})$ densities. The bounds in the density are in comparison with water density of $1.00 \mathrm{~g} / \mathrm{cc}$. Two different velocity regimes were chosen for this study to categorise the events as low-and highvelocity cases, where rebound occurred at the lower velocity and a flow-like behaviour occurred at the higher velocity.

The test matrix used for the present study is shown in Table 1. Impact generated due to bird strike on the test fixture is noted by measuring strain-time histories on the cylindrical hub part of the test fixture. The impact force created by the bird impact generates a stress wave that propagates along the cylindrical hub of the fixture. The finite element model used for the present study is shown in Fig. 4. The fixture was analysed to study the load $v s$ strains in the hub and the typical natural frequencies.

The fixture was intended to hold the leading edge of a typical transport aircraft and its dynamics is important in understanding the level of energy absorption by the leading edge, which will be reported separately. Here, the fixture is used to understand the effect of the bird impact on exciting the various frequencies of the fixture and the duration of the high- frequency and low-frequency excitations of the fixture. Modal analysis of the test fixture was carried out to identify the influential natural frequencies and mode

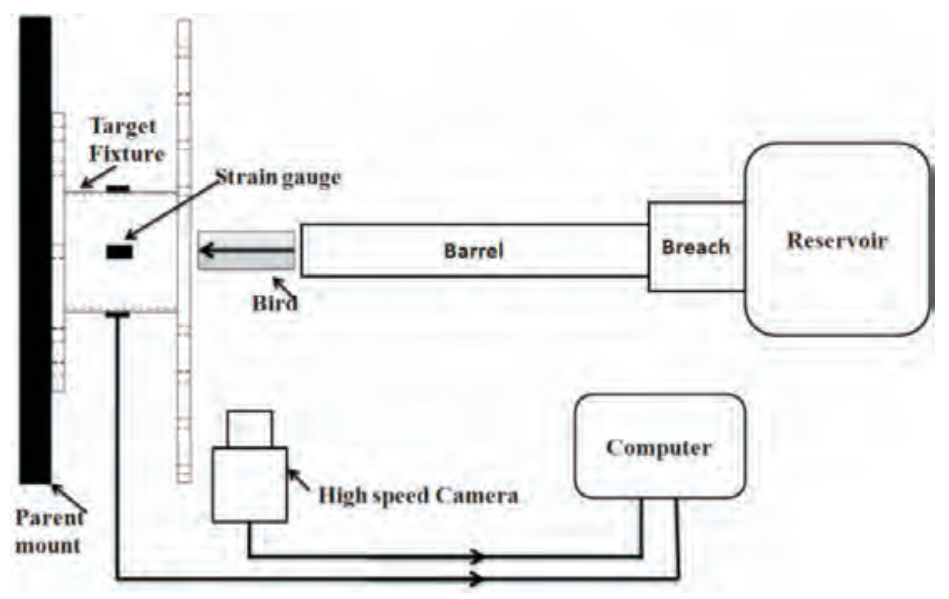

Figure 3. Experimental set-up used.

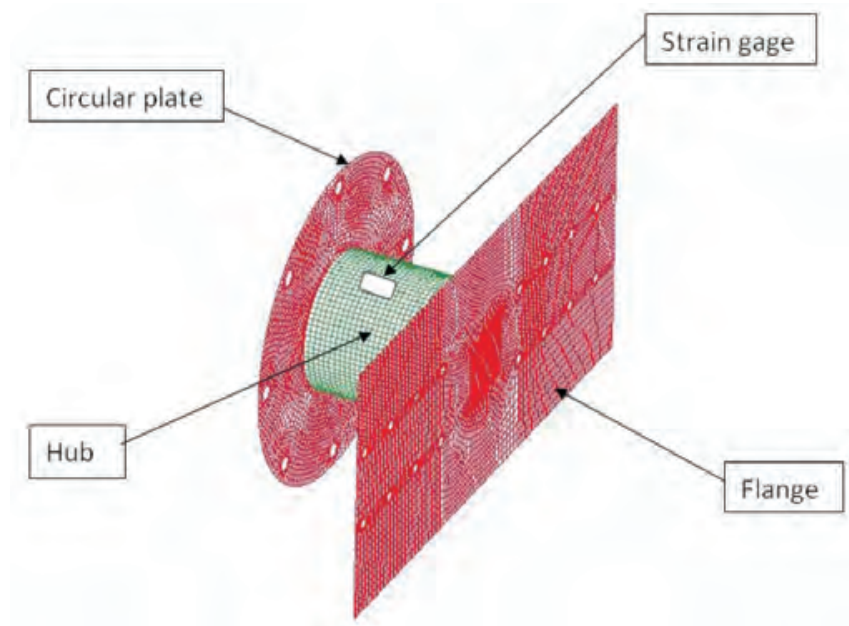

Figure 4. Target fixture finite element model used in the present study. 
Table 1. Test matrix used for present study.

\begin{tabular}{ccc}
\hline \multicolumn{3}{c}{ Case (a) } \\
Bird impact with low-velocity (rebound) \\
\hline Density (g/cc) & Bird mass (g) & Velocity $(\mathrm{m} / \mathrm{s})$ \\
\hline 0.9200 & 890 & 51 \\
0.9366 & 900 & 50 \\
1.1100 & 900 & 50 \\
\hline \multicolumn{3}{c}{ Bird impact with high-velocity (flow) } \\
\hline 0.9320 & 876 & 88 \\
0.9400 & 900 & 96 \\
1.1100 & 900 & 106 \\
\hline
\end{tabular}

shapes during bird impact ${ }^{14}$. From the modal analysis, it was noted that the frequencies span a wide range from $230 \mathrm{~Hz}$ to $3800 \mathrm{~Hz}$. This information should corroborate the types of excitations that occur at lower and higher velocities and can have an implication for local damage and impact loads that will occur on actual components of an aircraft. The mode shapes associated with these frequencies were also found and that shows a predominant vibration of the impacted plate. This vibration includes flexural, torsion, and axial modes. It is expected that even with very high target accuracies, small eccentricities during impact can excite all these modes. However, much of the higher modes are expected to remain only for a short period and these need to be examined from the signals. This is discussed further in the subsequent section.

\section{BIRD DEFORMATION BEHAVIOUR}

Bird mass of $0.909 \mathrm{~kg}$ was launched on the fixture at different velocities. The bird impact process were recorded through a high-speed camera to observe the impact, deformation, rebound (in some cases), and disintegration. During the test, it was made sure that the bird was intact and no change in orientation of the bird occurred prior to impact on the test fixture.

Image processing was carried out using Image Pro Plus Software ${ }^{15}$. The time histories of bird velocities were calculated by means of an automatic correlation-tracking procedure. The time histories of bird velocity measured from the high-speed image sequence recorded during the bird strike rebound test case (low-velocity) (a) is shown in Fig. 5. It shows the initial impact, compression, and rebound behaviour of bird from high-speed image sequences as well as respective velocities. It was observed that after the initial impact, the bird flew over the target and attempted to regain the original shape, but finally, it had distortions in both diameter as well as in length. The time histories of bird velocity measured from the high-speed image sequence recorded during the high-velocity bird strike is shown in Fig. 6. It shows the initial impact, flow and flow termination behaviour of bird from high-speed image sequences, as well as the respective velocities.

The time duration for the impact process till the bird spreads over the target, which is computed as noted $\mathrm{in}^{3}$ is dependent on the length of the cylinder and the velocity is consistent with the image processing done here.

Based on these tests, it can be seen that the bird behaves differently in case (a) low-velocity tests vis-avis case (b) high-velocity tests. It was observed that in the case (a), the cylindrical dummy bird impacted on the test fixture, compressed and expanded over nearly three times its original diameter in the radial direction. The behaviour was consistent with the description of an initial shock, radial spread (in the lateral direction perpendicular to the direction of impact and direction of flow (also see Fig. 5) and the entire cylinder flattened over the plate. Interestingly, a computation of the volume after the impact, before the bird reformed and rebounded versus the initial volume was noted, which indicated that at lower velocities, the release waves after the shock and the Hugoniot's shock pressures were not sufficient enough to cause the bird to flow completely as a fluid. Close examination of the high-speed image shows the effect of the sudden stoppage

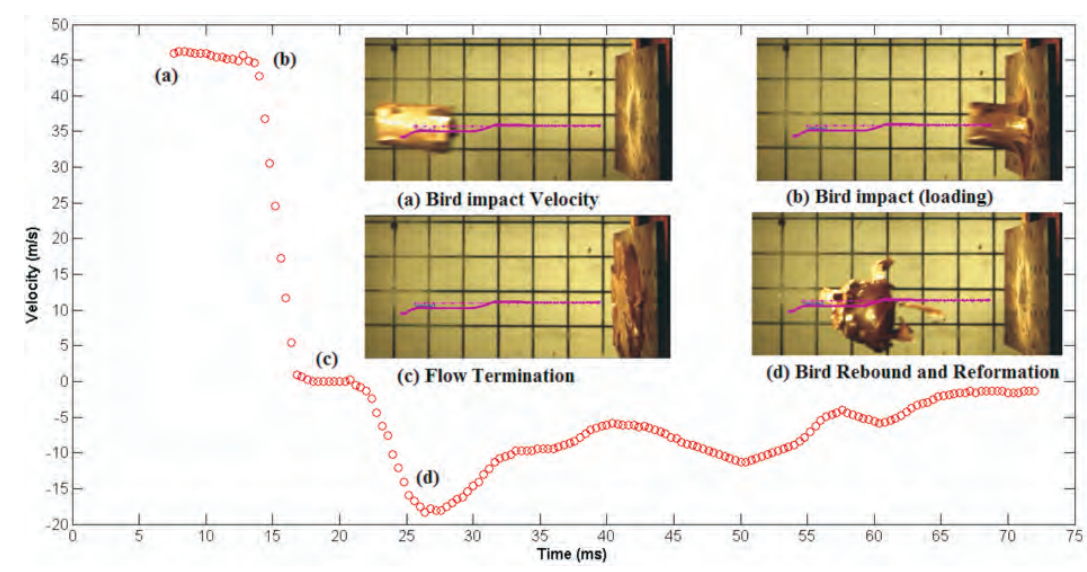

Figure 5. Time histories of velocity during low-velocity test using image processing.

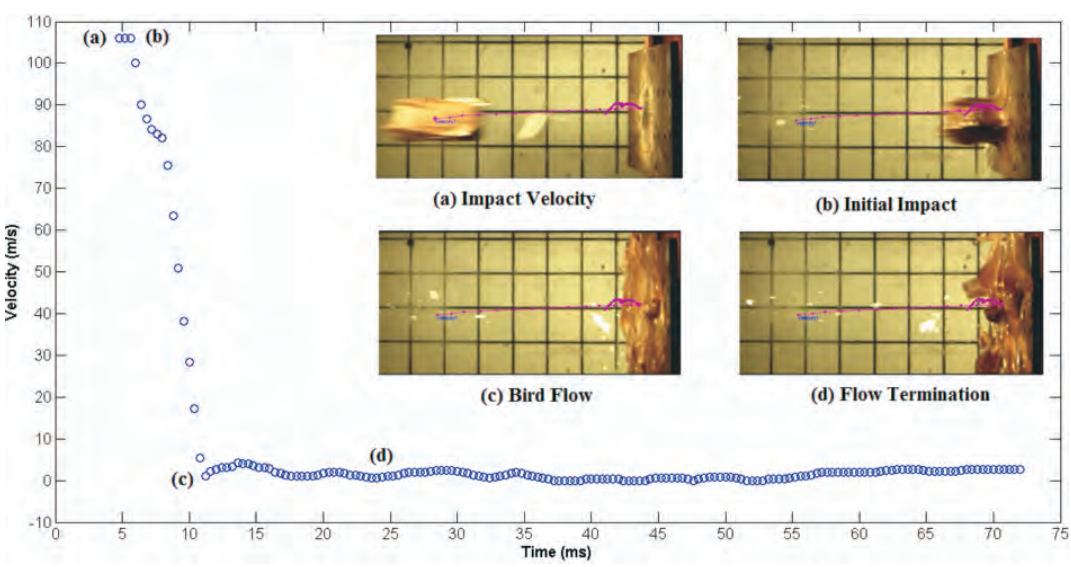

Figure 6. Time histories of velocity during high-velocity using image processing. 
of the bird. Here, while it did spread in the radial direction, it did not show the same texture that could be characterised as being a complete fluid as has been characterised at higher velocities. After the compression process, the bird attempted to regain its original shape. However this occurs with distortion in diameter and length. After the impact, the bird was in contact with the target plate for much longer and it took nearly $20 \mathrm{~ms}$ to reform and rebound. The bird appeared to store part of the kinetic energy during the compression process. A reformed bird that has the ability to rebound at lower speeds means that there is a need to use solid models with elasto-plastic and shear behaviour for simulations. The fact that residual energy was available for the bird to rebound was also a point to be noted in support of the solid rather than a fluid behaviour.

In the high-velocity impact case Fig. 6(b), the shock at the instant of impact was clearly seen. The bird now flows over the plate and is spread over the plate completely and even spills over. Here the radial spread is so rapid and large that it can clearly be characterised as fluid flow. It was observed from the sequence that the spread in the high-velocity case is much higher and the thickness of the bird after the spread is much lower than the lowvelocity case. However, the bird attempts to reform here as well, but with no ability to rebound and appears to have lost all energy. Here again, the duration of impact is consistent with the predictions associated with the length of the bird and the velocity of impact. Attempt to reform takes over $20 \mathrm{~ms}$. Since all energy has been lost, the duration in which load transfer takes place is also probably lower. The ability of the bird to retain some semblance of a solid, while flowing at impact requires to be noted. Modelling of the bird for simulations as a fluid is valid for high velocities, but post-impact behaviour requires to be carefully studied, especially from the perspective of including shear strength (see Figs 5 and 6 for details).

The behaviour of the bird in the low-velocity case raises issues with regard to the assumption that the bird behaves as a fluid as noted by Barber $^{3}$, et al. though in the high-velocity case, the impact process could be still considered as such. In the study by Barber ${ }^{3}$, et al. there have been no tests at the lower velocities. However, a number of transport aircraft tend to approach or take-off at about $50-65 \mathrm{~m} / \mathrm{s}$ and the behaviour of the bird at such velocities assumes significance. Simulation processes that are normally undertaken using LS-DYNA, PAMCRASH, ABAQUS, DYTRAN which are FE-based software and use models where the bird is modelled as a fluid (a ball/cylinder of water with a equation of state model (EOS)), or as a fluid in a Eulerian mesh (Arbitrary Euler-Lagrangian model), or as a smooth particle hydrodynamics SPH) mesh, produce varying results. It appears that the residual energy that is available as the bird reforms needs to be noted, as more sophisticated simulation models have been developed by the authors. This is even more important, if penetration occurs and components downstream need to be modelled for impact. In such situations, one will need to understand the behaviour of the bird in more detail.

\section{SIGNAL PROCESSING}

In the previous section, the bird deformation behaviour in two regimes of impact was highlighted. We now investigate the strain signals in the time and frequency domains have also been investigated. Wavelets were used to analyse frequencies and their disintegration over the impact period. These two signals were studied in detail to obtain the temporal and spatial relationships between the time and frequency domains. The results are indeed indicative of the usefulness of such techniques for locating frequency components over time. As a result, the time-frequency analysis is carried out for interpreting bird impact strain gauge signals in this study.

\subsection{Bird Impact Strain Signal Processing and Interpretation}

The time histories of the strain gauge signals at a typical location are shown in Figs. 7 and 8 for various
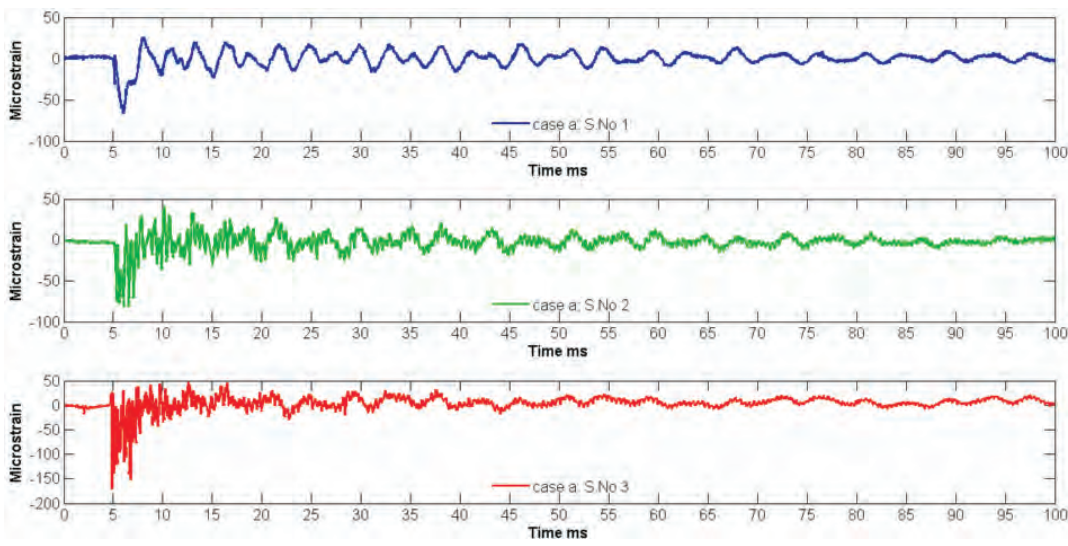

Figure 7. Time series strain signal measured for low-velocity (rebound) test case.
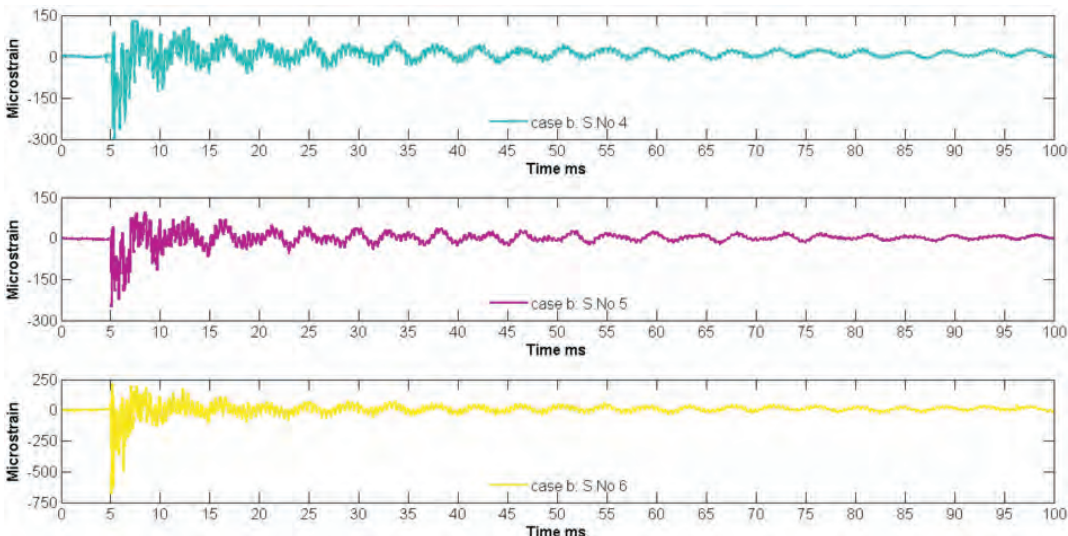

Figure 8. Time series strain signal measured for high-velocity (flow) test case. 

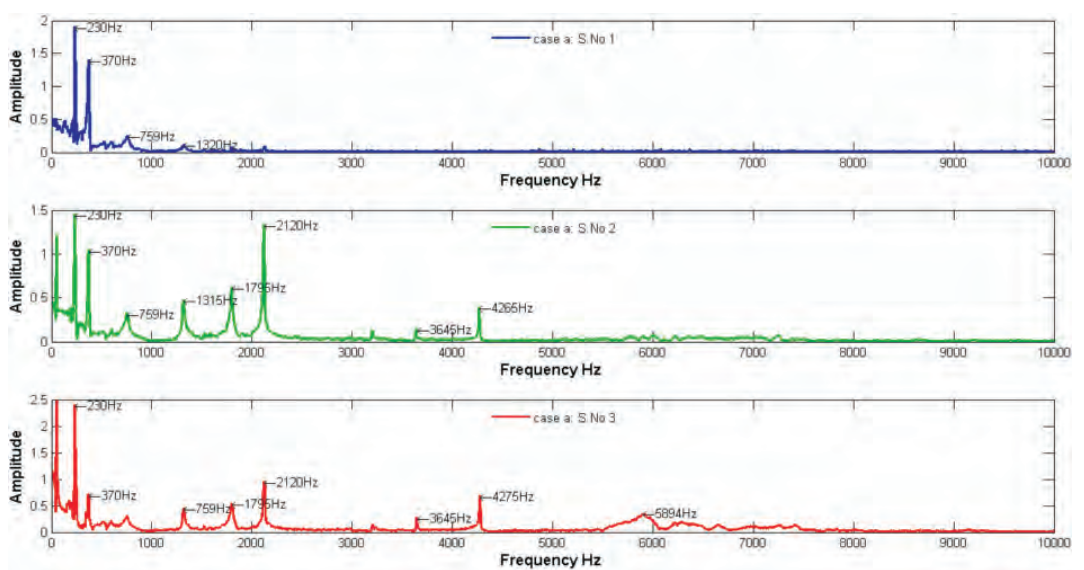

Figure 9. Fast fourier transform of strain signal measured for low-velocity (rebound) test case.
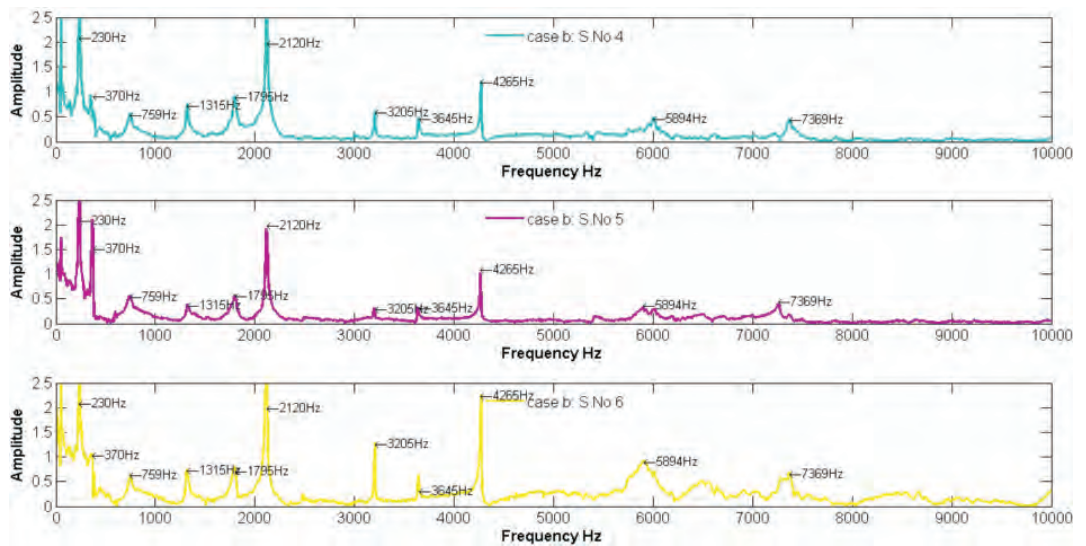

Figure 10. Fast fourier transform of strain signal measured for high-velocity (flow) test case.

speeds and bird densities. It is clear that the fixture shows a high frequency response at the time of impact. After the impact, the natural vibrations of the fixture are captured. It can also be seen that the strain response is higher at higher velocities.

As the density of the bird increases, its effect on the higher strains and dynamics of the fixture becomes clearly evident. Multiple frequency excitations are seen in the strain response during bird impact stage $(5-10 \mathrm{~ms})$. It is seen that increase of kinetic energy due to increase in velocity of the bird, increases impact loads by three-fold for the same bird density of $0.92 \mathrm{~g} / \mathrm{cc}$. Increase in density is also seen to increase the peak strain in both the test cases.

\subsection{Fast Fourier Transform Analysis}

A fast fourier transform of strain gauge signals was carried out to identify the frequency components excited during the bird impact process in the various test cases of the test matrix.

The high-velocity cases excite higher frequencies in the spectrum. The effect of density on the frequencies can be seen clearly as well. These signals show the effect of velocity on energy transferred to the fixture and also indicate the nature of frequencies that are excited. Many frequencies are reasonably close to that obtained in the finite element modal analysis of the fixture.

\subsection{Wavelets}

Generally, signal processing uses the Fourier method to extract the frequency information in the signal. If a signal contains a transient point of a finite time interval, its Fourier transform includes the contribution from the transient pulse, but the information about the transient point is lost on the time axis. Short time fourier transform (STFT) is sometimes used to obtain temporal as well as frequency axes resolutions. This however is not well-suited for the analysis of transient signals and in particular, to the generation of features for detection and discrimination. The accuracy of the STFT for extracting localised time/frequency information is limited by the length of this window relative to the duration of the signal. If the window is long in comparison to the signal duration, there will be time averaging of the spectral information in that window. On the other hand, the window must be long enough so that there is no excessive frequency distortion of the signal spectrum ${ }^{16}$. Crash-event signals are not stationary but transient and strongly localised in time. Consequently, conventional analysis used for stationary signals may not be appropriate or efficient for the analysis of crash signals ${ }^{17}$. Therefore, wavelets are used as a tool for signal analysis here, to study bird strike impact signals.

This scaling is a primary characteristic of wavelet analysis. It may be considered as a window function both in the time and in the frequency domains. The size of the time window is controlled by the translation while the length of the frequency band is controlled by the dilation. It is possible to change the size of the window either in time or frequency domain by controlling the extent of dilation and translation. This property of the wavelet transform is called multiresolution ${ }^{18.19}$. The Morlet wavelet is used as the analysing wavelet in this study, because it provides a small window in both time and frequency domains ${ }^{20}$. Benchmark studies were first carried out using the Morlet wavelet for both stationary and nonstationary signals. Stationary signals consist of a sinusoid with known frequencies whereas nonstationary signals consist of impulses created at specific time intervals in the sinusoid signals.

\subsection{Wavelet Analysis of the Signals}

The wavelet analysis is very useful in understanding the temporal issues wrt the frequencies being excited. In other words, one can clearly see that many high frequency responses occur only during the impact phase and 
subsequently, the natural frequency response takes over with the first few frequencies dominating. The wavelet analysis is also useful in identification of events. Wavelet analysis of strain signals obtained from the low-velocity (rebound) and high-velocity (flow) test cases are shown in Figs 11 and 12. These can be classified as the events based on the frequencies excited. The events are (a) impact (shock), (b) release, (c) rebound/loss of contact, and (d) natural vibration of fixture. This can be clearly seen in the form of three events. In Fig. 11 one can see three events, viz., Event E1-Bird impact and contact period,
Event E2-Bird rebound, and Event E3-Fixture first harmonic excitation. Similarly in Fig. 12, the events are marked as Event E1-Bird impact and contact period, Event E2-Bird flow period and Event E3-Fixture first harmonic excitation. The bird density is observed to exhibit an influence on the response, in higher frequencies and amplitudes.

Some distinct frequencies excited during the lowvelocity case (rebound) were noted. The behaviour of the bird as a fluid clearly encourages the higher frequency response in the high-velocity cases. In the low-velocity case, the frequencies excited were lower, with a band
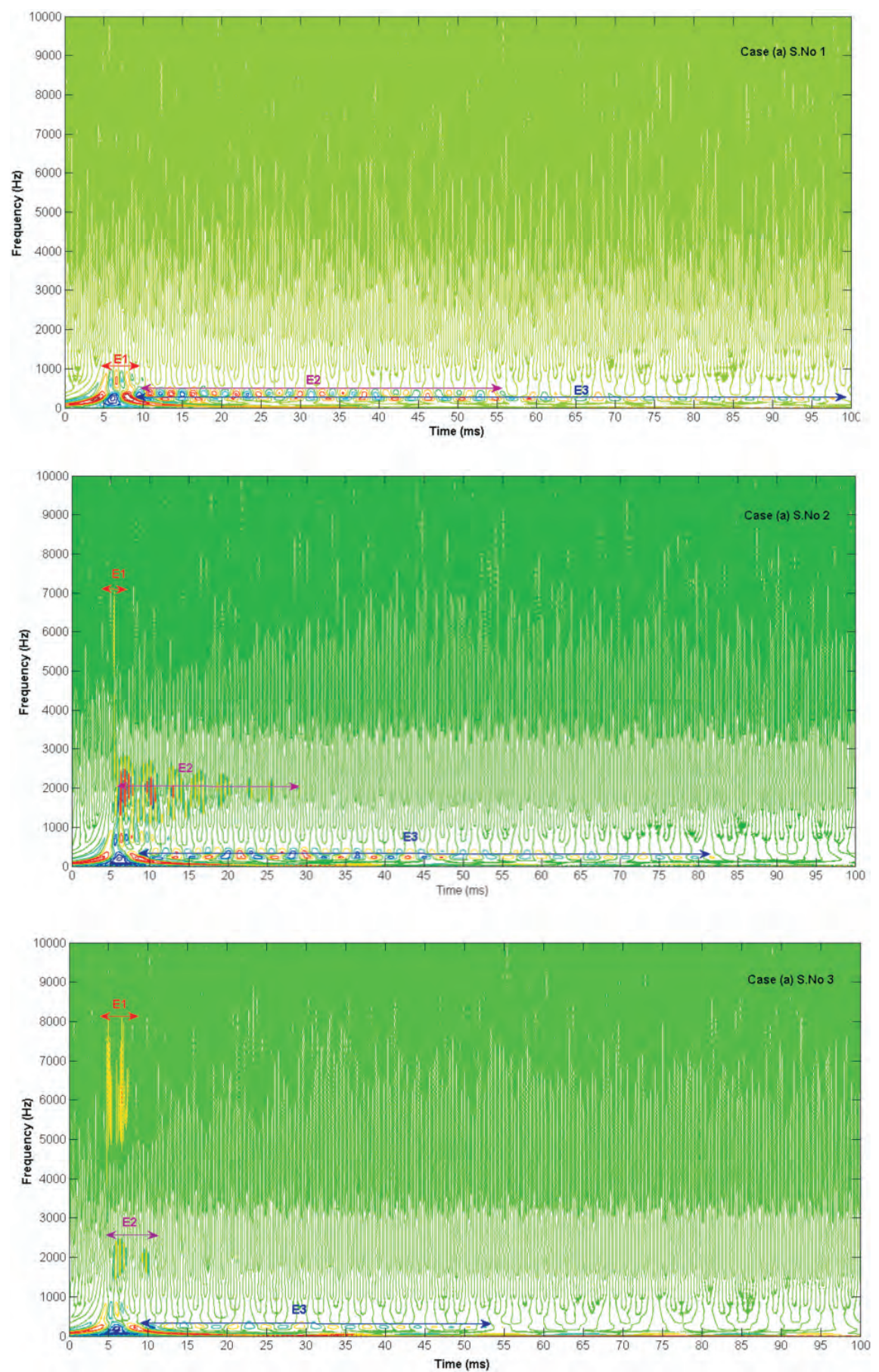

Figure 11. Wavelet analysis of bird low-velocity (rebound) test case strain signals. 

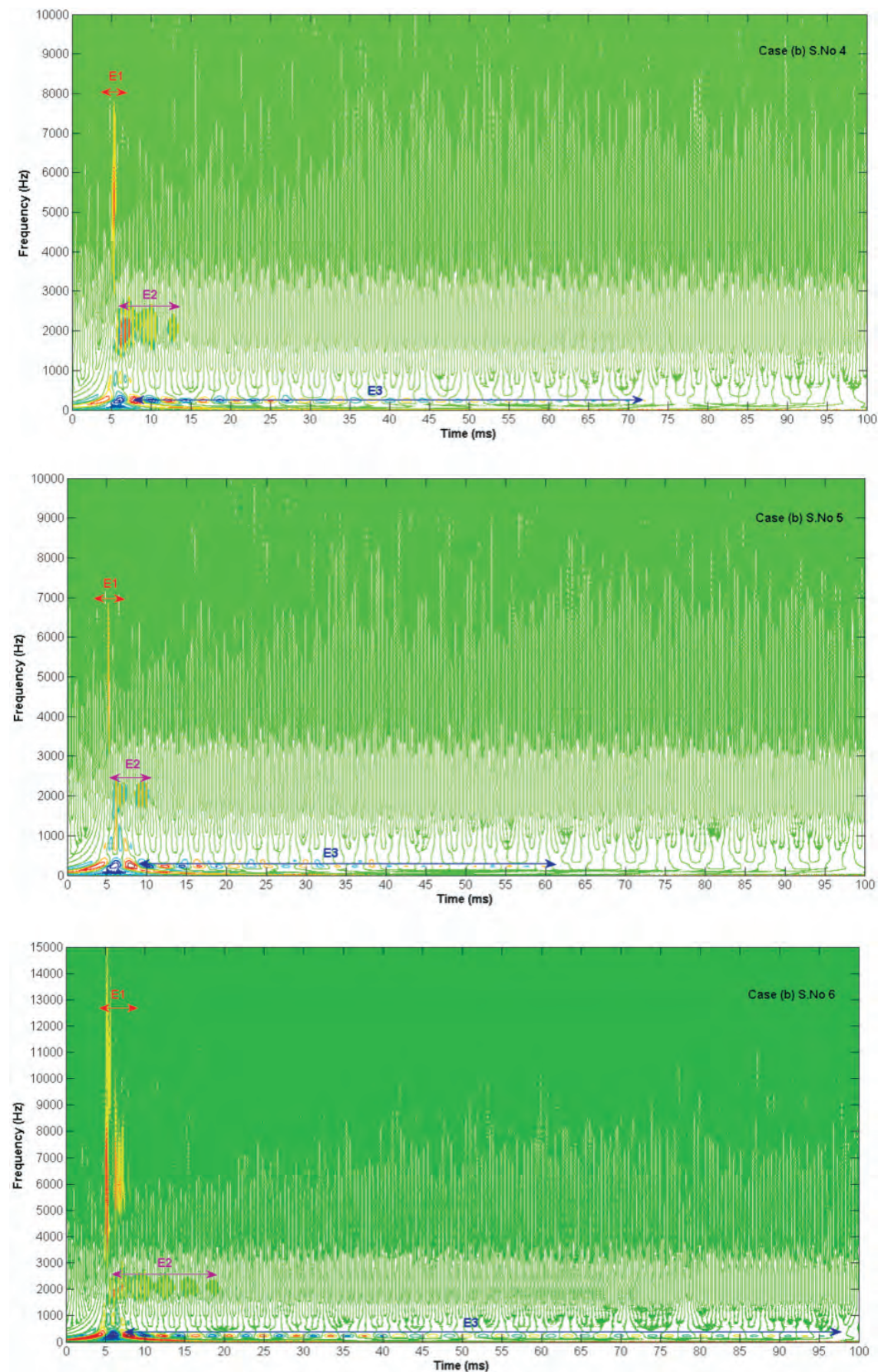

Figure 12. Wavelet analysis of bird high-velocity (flow) test case strain signals.

of $800-8000 \mathrm{~Hz}$, as seen from the FFT analysis. In the high-velocity case, frequencies of over $15000 \mathrm{~Hz}$ were excited. Further, as the density increases, one find that the frequencies seen during impact also tend to increase. Thus, both from the raw signals as well as the wavelets, one sees high frequency and higher strains with increase in density. In both the cases, the fixture settles down to vibrate at its natural frequency. Figures 13 and 14 show the wavelet processing localised to the impact duration. Event E1, which is the combination of loading and unloading is clearly split into two phases (1) and (2) in both the cases.
The wavelet analysis for the low-velocity case also shows the fact that while the duration of compression $(t)$ is reasonably consistent with the empirical formula $(t=L /$ $V)$ where $L$ is the length of the bird and $V$ the velocity, the bird remains in contact with the fixture till it reforms completely and then rebounds. This means that one can categorise two distinct phases of loading and unloading. Both these phases are seen in the high-speed imaging as well. The bird also tends to transfer loads for a longer duration. In the high-velocity case (Fig. 14), one can see that much higher frequencies are generated than in the low-velocity case. 


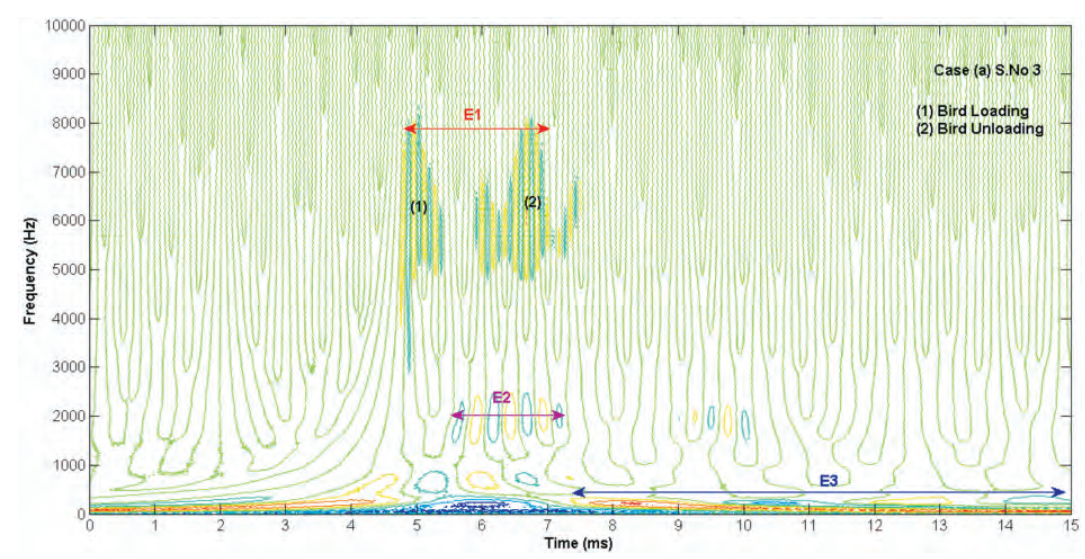

Figure 13. Loading and unloading of bird impact event in time-frequency analysis of strain gauge signal measured during low-velocity (rebound) test.

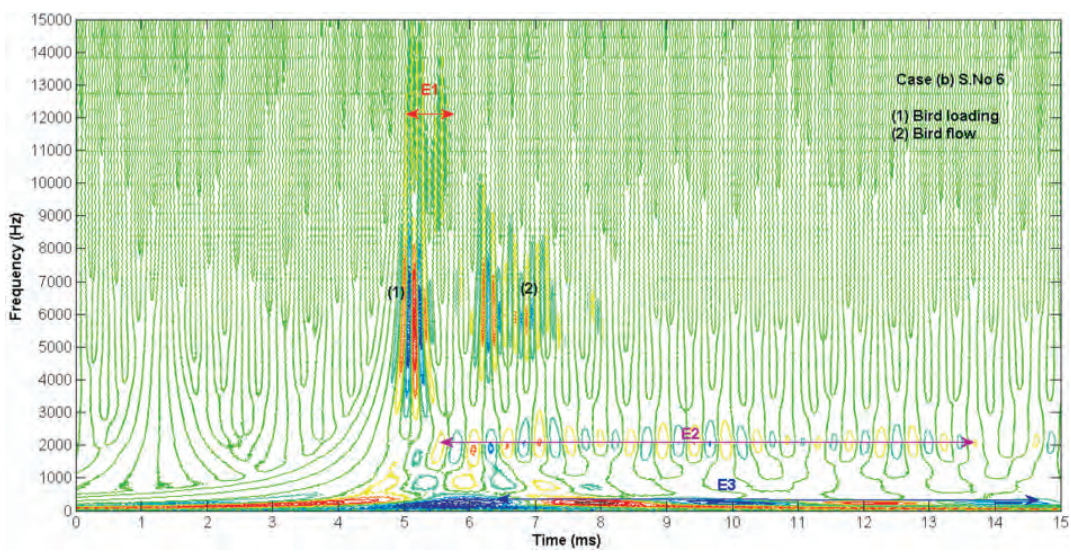

Figure 14. Loading and unloading of bird impact event in time-frequency analysis of strain gauge signal measured during high-velocity (flow) test. like a fluid, spreading over the fixture and thus could have exceeded its shear strength. Although the bird attempted to reform later, it failed and lost all its energy and did not rebound indicating near-fluid behaviour. However, its shear strength and related properties merits further investigation, which would become useful for simulation modelling.

Effect of the increase in kinetic energy was observed at higher speeds, by the processing of the strain signals. After impact, the natural vibration of the fixture could be seen. A Fourier transform clearly shows the frequency spectrum in which the bird impact events operate. In the higher-velocity case, the frequencies and amplitudes are higher. Wavelet analysis shows the duration and different phases of the impact event. Interestingly, the underlying low frequency fundamental mode of the fixture is seen throughout. Put together, these observations provide an insights useful for design of components resistant to bird strike based on the duration of impact, nature of the frequency spectrum encountered, and the type of fluid-/solid behaviour that exists.

\section{ACKNOWLEDGEMENTS}

The work was partly supported by Aeronautical Research and Development Board (AR\&DB). The support of the SIMA group at GTRE, Bengaluru in carrying out the bird strike tests is gratefully acknowledged. Test support from Mr Ganesh Kumar is also acknowledged.
The bird remains in contact for a larger period, but the loads are now spread over a larger area and the entire energy is transferred to the fixture. Here one sees that the duration of impact is lower, but high frequencies of the fixture continue as the bird remains in contact longer. From a design perspective, one can take note of the frequency components that exist in both low- and high-velocity impacts.

\section{CONCLUSIONS AND FUTURE RESEARCH}

The bird impact on a near rigid fixture has been detailed in this paper. Based on earlier work ${ }^{2-4}$, bird impact is known to be a short-duration, high-frequency event. However, it was noted that this event has different phases including shock, stagnation, and rebound/disintegration and it was observed that it behaves like a fluid which has led to a host of simulation models considering the bird as a fluid. In this study, the bird impact behaviour was examined using high-speed imaging and signal processing including a wavelet analysis. It was found that at lower speeds, after the initial shock, the bird spread in such a way indicating that shear stresses were within the limits and the bird reformed (albeit with distortion) and rebounded with the stored energy. At higher speeds, the bird effectively behaved

\section{REFERENCES}

1. Anon. Airworthiness Standards: Transport Category Airplanes, Federal Aviation Regulations (FAR), Part 25, Federal Aviation Administration, Washington, DC, USA, 2008.

2. Barber, J.P. \& Wilbeck, J.S. Characterization of bird impacts on rigid plate: Part 1, Air Force Flight Dynamics Laboratory, Technical Report No. AFFDL TR-75-05, 1975.

3. Barber, J.P.; Taylor, H.R. \& Wilbeck, J.S. Bird impact forces and pressures on rigid and compliance targets, Air Force Flight Dynamics Laboratory, Technical Report No. AFFDL TR-77-60, May 1978.

4. Wilbeck, J.S. Impact behaviour of low-strength projectiles, Air Force Flight Dynamics Laboratory, Technical Report, AFML TR-77-134, 1978.

5. Brockman, R.A. Finite element analysis of soft body impact. University of Dayton Research Institute. Report No. AFWAL-TR-84-3035, 1984

6. McNaughtan, I.I. The design of leading edge and intact wall structures to resist bird impact. Royal Aircraft Establishment. Technical Report No. 72056, 1972.

7. Johnson, A.F. \& Holzapfel, M. Modeling soft body 
impact on composite structure. Composite Structures, 2003, 61(1), 103-113.

8. Hanssen, A.G.; Girard,Y.; Olovsson, L.; Berstad, T. \& Langseth, M. A numerical model for bird strike of aluminum foam-based sandwich panels. Intl. J. Impact. Engg., 2006 32(7), 1127-144.

9. Kim, M.; Vahdati, M. \& Imregun, M. Aeroelastic stability analysis of a bird-damaged aero-engine fan assembly. Aero. Sci. Technol., 2001, 5(7), 469-82.

10. Airoldi, B. \& Cacchione, A. Modeling of impact forces and pressures in Lagrangian bird strike analyses. Intl. J. Impact. Engg., 2006, 32(10), 1651-677.

11. Yupu, Guan; Zhenhua, Zhao; Wei, Chen \& Deping, Gao. Foreign object damage to fan rotor blades of aero engine part I: Experimental study of bird impact. Chinese J. Aeronautics, 2007, 20(5), 408-14.

12. Hou, J.P. \& Ruiz, C. Soft body impact on laminated composite materials. Composites Part A: Appl. Sci. Manufacturing, 2007 38(2), 505-15.

13. Meguid, S.A.; Mao, R.H. \& Ng, T.Y. FE analysis of geometry effects of an artificial bird striking an aeroengine fan blade. Intl. J. Impact. Engg., 2008, 35(6), 487-98.

14. MSC Nastran, Analysis guide, MSC Software Corporation, USA 2007.

15. Image Pro Plus 6.2, Image analysis software, Media Cybernetics Inc.

16. Learned, R.E.; Karl, W.C. \& Willsky, A.S. Wavelet packet based transient signal classification, M.I.T, Report No. LIDS-2120, 2006.

17. Cheng, Z.Q. \& Pellettiere, J.A. Correlation analysis of automobile crash responses based on wavelet decompositions. Mechanical systems and signal processing, 2003, 17(6), 1237-257.

18. Strang, G. \& Nguyen, T. Wavelets and filter banks. Wellesley: Wellesley-Cambridge Press, 1996.

19. Y. Wu, R. Du, Feature extraction and. assessment using wavelet packets for monitoring of machining processes. Mechanical Systems and Signal Proces., 1996, 10(1), 29-53.

20. Lin, Jing \& Qu, Liangsheng. Feature extraction based on Morlet wavelet and its application for mechanical fault diagnosis. J. Sound Vibration, 2000, 234(1), $135-48$

\section{Contributers}

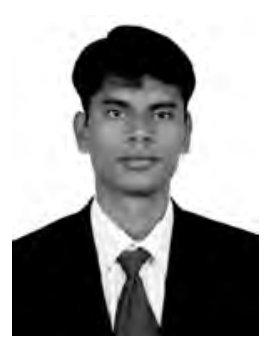

Mr Karthikeyan K. obtained MS (Aerospace Engineering) from Indian Institute of Technology Madras in 2004. At present, he is pursuing doctoral studies at Cambridge University Engineering Department. His research interests are composites, impact mechanics and digital image processing.

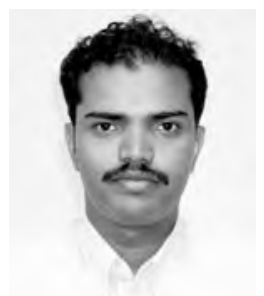

Mr Ramachandra holds a Masters degree in Mechanical Engineering from IISc and is currently working as Scientist ' $F$ ' at Gas Turbine Research Establishment (GTRE), Bengaluru. He has over 23 years experience in the field of impact mechanics, structural analysis and testing of aero engine components. He has more than 35 publications in International Journals and Conferences. He is Fellow of Institution of Engineers and Institution of Production Engineers. He has worked for certification programs of Kaveri engine, and other major national projects, viz. ALH, LCA, SARAS aircraft and AEW\&C. His other fields of activities include high temperature PMC's and CMC's for aero engines.

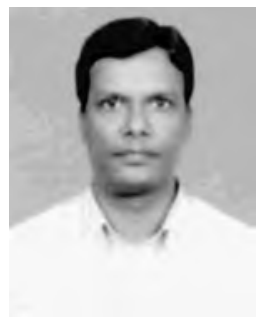

Dr S. Paul Vizhian is currently a Professor of Mechanical Engineering at the University Visveswaraya College of Engineering, Bengaluru. He obtained his Bachelor, Masters and PhD from the University of Bengaluru. He is Fellow of Institution of Production Engineers and life member of Institution of Engineers and. ISTE. He has more than 25 years of academic experience. He has more than 50 publications in National and International Conferences and Journals. His main areas of interest are experimental mechanics and composites.

Dr Satish Chandra is presently the Project Director, NCAD and is holding additional charge of Head, Structural Technologies Division, at National Aerospace Laboratories and specialises in impact and crashworthiness. He has a $\mathrm{PhD}$ from the University of Bristol, England. He has published over 100 papers and technical reports. He has over 25 years of experience in the field. 